\title{
Compartilhamento do conhecimento na melhoria da dinâmica organizacional em um serviço de teleterapia
}

Sharing knowledge in improving organizational dynamics in a teletherapy service

\author{
P. F. Dorow ${ }^{1 *}$; D. F. J. Ramos ${ }^{2}$; C. D. Medeiros ${ }^{1}$; T. S. C. Camozatto ${ }^{1}$; C. D. \\ Silva $^{3}$; F. C. Vargas ${ }^{3}$; C. Salvador ${ }^{3}$; A. Huhn ${ }^{1}$; P. M. Rodrigues ${ }^{4}$ \\ ${ }^{I}$ Departamento Acadêmico de Saúde e Serviços, Curso Superior de Tecnologia em Radiologia e Mestrado Profissional \\ em Proteção Radiológica, Instituto Federal de Santa Catarina, 88020-300, Florianópolis-Santa Catarina, Brasil \\ ${ }^{2}$ Departamento Acadêmico de Saúde e Serviços, Curso Superior de Tecnologia em Radiologia, Instituto Federal de \\ Santa Catarina, 88020-300, Florianópolis-Santa Catarina, Brasil \\ ${ }^{3}$ Departamento Acadêmico de Saúde e Serviços, Mestrado em Engermagem, Instituto Federal de Santa Catarina, \\ 88020-300, Florianópolis-Santa Catarina, Brasil \\ ${ }^{4}$ Centro de Pesquisas Oncológicas, Rodovia Admar Gonzaga, Itacorubi, 88034-000, Florianópolis - SC, Brasil \\ *patriciad@ifsc.edu.br \\ (Recebido em 17 de agosto de 2018; aceito em 12 de janeiro de 2019)
}

\begin{abstract}
$\mathrm{Na}$ área da saúde, principalmente na radioterapia, o trabalho em equipe exige eficiência e coordenação, ou seja, a gestão do conhecimento, principalmente, o compartilhamento do conhecimento torna-se ainda mais necessário para garantir a qualidade na entrega do tratamento. Segundo a literatura, as equipes de trabalho coesivas em radioterapia possuem cinco características fundamentais: metas claras com resultados mensuráveis, sistemas clínicos e administrativos apropriados, divisão do trabalho, treinamento de todos os membros da equipe e comunicação efetiva. A presente pesquisa teve como objetivo geral analisar o compartilhamento do conhecimento na melhoria da dinâmica organizacional em um serviço de teleterapia. Para tanto, realizou-se uma pesquisa qualitativa por meio de entrevistas semiestruturadas com os profissionais envolvidos no funcionamento de um serviço de radioterapia da grande Florianópolis. Participaram do estudo seis profissionais. Para análise dos dados foi utilizada a técnica de análise de conteúdo. Os resultados indicam que, em relação ao compartilhamento do conhecimento no local pesquisado, alguns fatores identificados como a comunicação efetiva, melhoram a dinâmica organizacional envolvida no cuidado com o paciente oncológico, sendo esse um dos fatores que contribuem para que a entrega da dose real seja a mais próxima possível da entrega da dose prescrita para o tratamento. As principais práticas de compartilhamento do conhecimento identificadas foram: revisão por pares, mentoring e storytelling. Conclui-se que os fatores organizacionais responsáveis por melhorarem o compartilhamento do conhecimento como o uso das estratégias evidenciadas neste estudo ajudam na garantia da reprodutibilidade diária do tratamento, consequentemente, na melhora da qualidade de vida do paciente oncológico.

Palavras-chave: Análise de conteúdo, Compartilhamento do Conhecimento, Radioterapia, Reprodutibilidade.
\end{abstract}

In the area of health, especially in radiotherapy, teamwork requires efficiency and coordination, that is, knowledge management, especially, sharing of knowledge becomes even more necessary to guarantee the quality of treatment delivery. According to the literature, cohesive work teams in radiotherapy have five key characteristics: clear goals with measurable outcomes, appropriate clinical and administrative systems, division of labor, training of all team members, and effective communication. The present research had as general objective to analyze the knowledge sharing in the improvement of the organizational dynamics in a teletherapy service. Therefore, a qualitative research was conducted through semi-structured interviews with the professionals involved in the operation of a radiotherapy service in the greater Florianópolis. Six professionals participated in the study. To analyze the data, the content analysis technique was used. The results indicate that, in relation to the sharing of knowledge at the place of study, some factors identified as effective communication improve the organizational dynamics involved in the care of the cancer patient, being one of the factors that contribute to the delivery of the actual dose as close as possible to the delivery of the dose prescribed for treatment. The main practices of knowledge sharing identified were: peer review, mentoring and storytelling. It was concluded that the organizational factors responsible for improving the knowledge sharing as the use of the strategies evidenced in this study help to guarantee the daily reproducibility of the treatment, consequently, to improve the quality of life of cancer patients.

Keywords: Content analysis, Sharing Knowledge, Radiotherapy, Reproducibility. 


\section{INTRODUÇÃO}

O aumento de novos casos de câncer, o alto custo, a complexidade das tecnologias para tratamentos de radioterapia e o contingenciamento de verbas para a saúde justificam pesquisas para um compartilhamento do conhecimento efetivo. Os maiores casos de incidentes de tratamento com radiação estão relacionados a erros humanos [1]. A radioterapia consiste em uma dessas formas de tratamento que utiliza um sistema complexo, dada a coexistência de elementos variáveis como a natureza particular da alta energia de radiação utilizada, a necessidade de trabalho em equipe entre profissionais de diferentes especialidades e o forte aspecto técnico que evolui continuamente [2].

Dessa forma, todos os profissionais que fazem parte da equipe multidisciplinar (médicos, enfermeiros, tecnólogos em radiologia, físicos, entre outros) envolvidos no processo radioterápico devem executar os procedimentos com cautela para garantir a reprodutibilidade do tratamento de forma precisa e segura. $\mathrm{O}$ trabalho da equipe multidisciplinar é, então, considerado muito importante pelos serviços de saúde e atualmente está amplamente estabelecido em todas as esferas da prática médica. Nos dias atuais, para se tratar um paciente com sucesso em radioterapia é necessária uma tecnologia especializada e uma integração complexa entre os membros que formam a equipe multidisciplinar [3].

Nos serviços oncológicos, esse trabalho entre a equipe é vital para o bom funcionamento da prática oncológica. Além disso, qualidade em radioterapia é um conceito dinâmico que precisa ser medido e reavaliado usando métodos científicos e feedback dos usuários. A execução bem sucedida de um programa de garantia de qualidade em radioterapia requer experiência, treinamento e coordenação em um ambiente de trabalho em equipe [4].

Aliado a isso, tem se que a utilização do conhecimento vem crescendo por ser esse, um recurso que permite as organizações de saúde possa planejar e otimizar suas ações para obter resultados mais eficazes [4]. Dessa forma, a gestão da experiência, competência e conhecimento em relação aos processos de trabalho e melhores práticas são importantes [6]. Sendo assim, o compartilhamento do conhecimento é um dos processos que permite o crescimento do conhecimento, pois permite que as organizações possam explorar e capitalizar os recursos baseados no conhecimento para a melhoria do desempenho organizacional. $\mathrm{O}$ acesso rápido dos profissionais ao conhecimento dentro da organização permite agir rapidamente e assertivamente em relação às decisões do dia a dia [7].

No entanto, a literatura não explora como o compartilhamento do conhecimento acontece, quais as práticas utilizadas pelos indivíduos e como elas auxiliam no alinhamento do entendimento da equipe sobre fatores que envolvem o tratamento do paciente oncológico.

Karamitri et al. (2015) [8] realizaram uma extensa revisão sistemática da literatura de práticas de gestão do conhecimento em contextos de saúde e concluíram que os estudos futuros devem centrar-se em contextos de saúde, na identificação das melhores práticas e nas ações que promovem uma mudança comportamental dos indivíduos para compartilhar conhecimento. Velloso et al. (2015) [9] corroboram que a gestão do conhecimento, especificamente o compartilhamento do conhecimento, influencia no comportamento dos colaboradores que atuam no setor da saúde e na forma como esses prestam atendimento aos pacientes.

Além disso, nas configurações médicas em que os médicos e outros profissionais trabalham juntos como equipes melhores resultados são fornecidos ao paciente. Entretanto, existem várias barreiras à formação de equipes, principalmente relacionadas aos desafios das relações e personalidades humanas. Estudos que ajudam a avançar em direção ao desenvolvimento das equipes podem melhorar o ambiente de trabalho nas práticas de radioterapia. Sendo assim, o objetivo desta pesquisa foi analisar o compartilhamento do conhecimento na melhoria da dinâmica organizacional em um serviço de teleterapia.

\section{MATERIAL E MÉTODOS}

Trata-se de pesquisa metodológica qualitativa, de caráter descritivo, que utilizou seis entrevistas semiestruturadas para coletar os dados. Para análise dos dados foi utilizada a técnica de análise de conteúdo de Bardin (2016) [10]. O trabalho encontra-se com aprovação do comitê de ética em pesquisa (CEP) pela Plataforma Brasil sob o parecer $n^{\circ} 15.806$. 
A pesquisa foi realizada em uma instituição privada de radioterapia da grande Florianópolis, sul do Brasil. Para compor a amostra foi escolhido um profissional de cada área que compõem a equipe multidisciplinar envolvida no tratamento de radioterapia: 01 médico oncologista, 01 físico-médico, 01 tecnólogo em radiologia, 01 técnico em radiologia, 01 enfermeiro e 01 dosimetrista. Para a escolha dos participantes do estudo utilizou-se a amostragem não probabilística intencional. Os critérios utilizados para seleção dos sujeitos foram: integrar a equipe multidisciplinar, atuar no mínimo há 3 anos no local pesquisado e concordar em participar do estudo assinando o Termo de Consentimento Livre e Esclarecido (TCLE).

A metodologia compreendeu quatro etapas. A primeira etapa consistiu na pesquisa bibliográfica, organização do material a partir de buscas em referenciais teóricos sobre o assunto abordado e síntese do material encontrado para definir o problema a ser pesquisado e demonstrar a importância da pesquisa. Na segunda etapa foi definido o instrumento de coleta dos dados, que consistiu na construção do questionário utilizado nas entrevistas semiestruturadas que norteou a pesquisa. $\mathrm{O}$ questionário foi composto de 31 perguntas, sendo seis para o radioterapeuta, sete para o físico, seis para o tecnólogo, seis para o dosimetrista e seis para o enfermeiro.

$\mathrm{Na}$ terceira etapa foram aplicadas as entrevistas semiestruturadas que ocorreram no período de fevereiro a abril de 2018. O processo de abordagem para a realização das entrevistas incluiu o contato prévio com o local de pesquisa e com os profissionais de saúde para breve apresentação da pesquisadora e dos objetivos da pesquisa, bem como o agendamento para a coleta de dados.

A quarta etapa consistiu na análise das entrevistas. O tempo médio de duração de cada entrevista foi de 20-30 minutos, sendo realizada no local de trabalho dos participantes. As entrevistas foram registradas por meio de gravação em áudio e transcritas na íntegra para facilitar a análise dos depoimentos. Para garantir o sigilo das falas foram criados codinomes de identificação para cada participante como segue: a) Médico Rádio-oncologista (E1); b) Dosimetrista (E2); c) Enfermeiro em Radioterapia (E3); d) Tecnólogo em Radiologia (E4); e) Técnico em Radioterapia (E5); f) Físico Médico (E6).

Para análise das entrevistas foi utilizada a técnica de análise de conteúdo de Bardin (2016) [10] que preconiza três etapas: a) pré- análise; b) exploração do material; e c) interpretação e discussão dos resultados.

Na primeira etapa pré-análise o material é preparado para a análise em si. Os dados coletados foram arranjados em um documento para conseguir um entendimento significativo, visando a obter dados suficientes e representativos para alcançar os objetivos propostos. Foram escolhidas as informações que definiram o corpus de análise e a formulação das unidades de análise para estabelecer a interpretação final.

$\mathrm{Na}$ segunda etapa foram explorados os dados brutos provenientes das entrevistas e, depois de transformados em unidades de análise, estes foram lidos e relidos para criação das características pertinentes ao conteúdo expresso. As categorias da pesquisa surgiram após leituras e releituras do tema, onde os temas norteadores (categorias) emergiram da repetição da mesma informação por diferentes participantes.

$\mathrm{Na}$ terceira etapa, interpretação e discussão dos resultados, buscou-se destacar as informações da análise e exibir o resultado final do estudo. Os dados significativos e fidedignos encontrados foram analisados de acordo com a literatura sobre o tema possibilitando interpretações por frequência de ocorrência.

\section{RESULTADOS E DISCUSSÃO}

O local pesquisado realiza diariamente cerca de 35 tratamentos com radioterapia de diferentes tipos e conta com um acelerador linear 600CD com energia de $6 \mathrm{MeV}$ da marca Varian Medical Sistems ${ }^{\circledR}$ dotado de um Microcolimador Multilâmina $\mathrm{m} 3$ da Brainlab® possibilitando a modelagem de feixe de alta resolução. Além disso, o sistema de radioterapia guiada por imagem ExacTrac ${ }^{\circledR}$ também da fabricante Brainlab® assegura precisão milimétrica nos tratamentos de radioterapia, radiocirurgia craniana, radiocirurgia corpórea e tratamentos hipofracionados com localização estereotáxica [11]. 
Por meio da análise do discurso dos participantes em relação as formas utilizadas para compartilhar conhecimento entre os profissionais que compõem a equipe multidisciplinar foram identificadas as seguintes unidades de análise: a) Interações face a face; b) Reunião presencial quinzenal; c) Reuniões virtuais; d) Grupo no WhatsApp; e) Mesas redondas; e f) Capacitações.

A Figura 1 apresenta a dinâmica existente entre as estratégias utilizadas pelo local estudado para compartilhar o conhecimento sobre o tema.

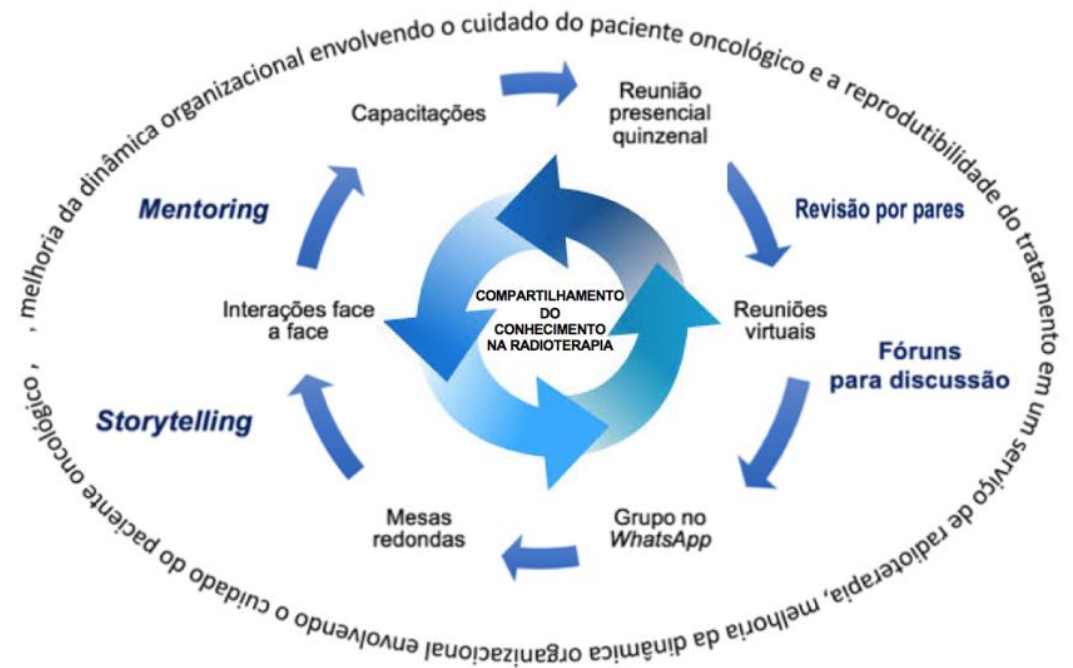

Figura 1 - Dinâmica do compartilhamento de conhecimento do local pesquisado.

Interações face a face: interações realizadas durante a rotina de trabalho. Segundo alguns entrevistados:

\footnotetext{
"a gente está sempre se comunicando para que todo mundo fale a mesma língua. Aqui na clínica o legal é que existe um padrão, não é cada um trabalha do seu jeito, todo mundo segue uma rotina, um padrão". E4

"temos processos operacionais voltados ao compartilhamento, mas é no próprio dia a dia que acontecem a maior frequência de interações, as especificidades de cada paciente são discutidas, mesmo que seja brevemente, ninguém fica com dúvida essa é uma cultura aqui dentro. A partir do momento que se vai planejar um tratamento de um paciente, todos estarão envolvidos". E1
}

Outro entrevistado complementou:

"estamos em constante conversa aqui, temos bom relacionamento, e qualquer mudança ou qualquer coisa que seja diferente, que saia da nossa rotina a gente está sempre anotando, de diversas maneiras, na ficha, na folha de rosto, e sempre passando uma para outra ou, as vezes, até alguma anotação na própria agenda, quando for chamar esse paciente para reforçar, então a gente sempre se comunica bem, assim.” E4

Percebe-se, por exemplo, na fala do entrevistado E1, a existência de um ambiente organizacional adequado, onde os indivíduos possuem um entendimento comum. As pesquisas de Wang e Noe (2010) [12] indicam que fatores como uma estrutura voltada ao compartilhamento do conhecimento, uma linguagem comum, uma cultura e um clima organizacional saudável favorecem o compartilhamento do conhecimento. Estes autores afirmam ainda que a confiança é fruto do bom relacionamento e como o compartilhamento do conhecimento é um comportamento voluntário, quando existe um ambiente organizacional adequado as interações são mais efetivas. Nas falas dos entrevistados percebe-se um grande comprometimento de toda equipe em ajudar uns aos outros, portanto existe uma cultura favorável a aprendizagem no local do estudo.

Os entrevistados adicionaram ainda a rotina corrida com os pacientes devido ao grande número de tratamentos, comentaram sobre a pressão que o médico oncologista sofre devido ao cuidado e atenção que exige esse trabalho. Devido ao fato do câncer ser um alvo social complexo, algumas 
pessoas não aceitam bem a doença e, dessa forma, a equipe multidisciplinar deve também fornecer suporte emocional tanto ao paciente quanto aos familiares.

Reunião presencial quinzenal: ocorre nas quartas-feiras. Segundo os participantes são muito ricas, envolvem discussões de casos, bem como conhecimento científico sobre biologia e oncologia. Segundo os entrevistados:

\footnotetext{
"a maior parte das reuniões é de temas pra aprofundamento científico nosso. Algumas são dadas pelos médicos, outras pelos físicos e pelos enfermeiros também, no dia-a-dia, se surge alguma coisa que a gente já pode falar, a gente já antecipa, mas são reforçadas sempre nas reuniões quinzenais". E3

"São discutidos casos ou quando tem alguma tecnologia nova ou até uma rotina diferente a gente costuma passar em reunião que pra todos fiquem sabendo dessas mudanças, dessa nova rotina." $\mathrm{E} 4$
}

Outro entrevistado complementa:

\begin{abstract}
“A pessoa que faz faxina na clínica participa da reunião também. Ela tem que saber o que está acontecendo, tem que ter uma noção básica e muito bem consolidada por que ela é o interlocutor frequentemente de alguma pessoa. E o que é passado como informação é aceito como verdade, queira ou não queira.". E1
\end{abstract}

A fala anterior do entrevistado 1 explicita que o que é passado como informação é aceito como verdade, e isso corrobora a ideia de conhecimento de Platão de que conhecimento são crenças verdadeiras e justificadas, ou seja, aspectos imprescindíveis para que se conheça algo [13].

Segundo os entrevistados, os encontros quinzenais são uma forma muito eficiente de compartilhar conhecimento, pois é um momento que todos estão juntos e dedicados a aprender uns com os outros. Nessas reuniões são tratados desde assuntos administrativos até assuntos técnicos, essa prática de fazer reuniões existe desde que a clínica foi fundada em 1978. O local pesquisado possui uma filial em Lages e nas quintas-feiras ocorrem essas reuniões, bem como mesas redondas que geralmente são as quartas ou quintas à noite. Percebe-se que existe uma grande preocupação com a rotina do paciente. Segundo Nambisan (2002) [14], reuniões são uma ótima forma de compartilhar conhecimento e estreitar as relações de empatia e confiança entre o grupo.

Percebe-se que todos que trabalham no local pesquisado, independente da função, interagem frequentemente principalmente na prática diária de trabalho e, mais tarde, a troca de conhecimentos é complementada por meio das reuniões, sendo que o fluxo de trabalho é feito para isso acontecer.

Reuniões virtuais: são reuniões realizadas por teleconferência e existem vários pólos que participam dessas reuniões. Segundo o entrevistado:

\footnotetext{
"Isso é uma constante, nós temos reuniões aqui há mais de 30 anos, no que se diz respeito ao conhecimento técnico e funcionamento da clínica, nós temos reuniões que são semanais e eventualmente quinzenais e que todos participam". E1
}

O entrevistado explicou que cada profissional que compõem a equipe multidisciplinar envolvida no tratamento possui necessidades específicas de conhecimento e, muitas vezes, os assuntos são variados. Para Rissi (2013) [15], as interações virtuais apresentam melhor resultado quando o grupo já possui laços afetivos e relações prévias de confiança.

Grupo no WhatsApp: utilizado para discussões, principalmente envolvendo o planejamento de alguns casos específicos. Conforme explica uma das participantes:

\footnotetext{
"Como temos a clínica de Lages e os médicos se revezam um pouco aqui, um pouco lá, então tem planejamentos que o físico faz, e ele precisa repassar alguma coisa, e o médico está em Lages. Então coisas do tipo: olha Dr. fiz o planejamento, deu dose de tanto no cristalino, mandou uma foto do planejamento, mandou um vídeo ou eu mandei uma foto de uma ressonância, Dr. olha essa paciente, então a gente acaba discutindo casos por meio desta ferramenta." E2
}

Nesse sentido, quando as pessoas desenvolvem confiança uma nas outras, criam um vínculo que leva à comunicação acidental porque a confiança entre as pessoas amplia o compartilhamento do conhecimento [16]. 
Mesas redondas: podem ser de neurologia, urologia, mama, dentre outras. São grupos de estudos, que se reúnem periodicamente, formados por diferentes profissionais de áreas como imagem, patologia, clínica e cirurgia, nesses grupos são discutidos temas específicos e cada profissional apresenta a sua expertise para contribuir com a discussão.

Capacitações: sempre que um profissional vai a algum evento (congressos, cursos) quando retorna tudo que foi aprendido é repassado aos colegas, conforme fala da entrevistada:

"Não é só a gente vai lá, dá o curso, repassa o conhecimento, mas a gente aprende muito
com isso. Então eu repasso aqui para o pessoal, os meus colegas como foi minha
experiência. Foi uma experiência única, foi uma experiência nova, diferente, um desafio,
e é muito bom, eu gosto bastante, no dia-a-dia também a gente troca conhecimento,
aprende.” E2

Já outro entrevistado tem uma visão diferente dos congressos para ele:

"os congressos são pra consolidar parcerias e ou dar uma aula. Para mim ir em um congresso fora do Brasil é mais interessante" E6

Pesquisas indicam que congressos, simpósios e encontros entre profissionais da área são uma ótima maneira de fazer networking, pois a colaboração entre diferentes organizações favorece a competitividade e impulsiona a inovação [17].

Aulas: compartilhamento do conhecimento mediado por meio de aulas expositivas realizadas pelos profissionais que detém algum conhecimento de interesse para os outros colegas.

\footnotetext{
“eu sigo listas de e-mail de discussão, eu tenho grupo grande de 309 físicos, eu participo de grupo de medicina nuclear que tem um monte de livros, discussão no mundo, eu tenho uma empresa de condicionamento na America latina, eu tenho uma empresa de software, então eu faço bastante coisa nesse sentido, eu estou sempre lendo, aprendendo, fazendo e conversando". E6
}

Em relação à atualização de conhecimentos, um dos entrevistados explicou:

\begin{abstract}
"eu acordo às 5 da manhã para estudar todos os dias até as 6:30, esteja aonde estiver, desde que me formei em médico e olha que eu vou fazer 50 anos de medicina, isso é diário, isso é um mantra que não vai deixar de acontecer nunca" E1
\end{abstract}

O entrevistado relatou que compra livros de oncologia, cancerologia e radioterapia a cada dois anos. Explicou que é necessário que se tenha um eixo formador de pensamentos e fragmentos. Hoje existem sites a disposição para consulta e alguns que efetivamente são referência. $O$ local pesquisado possui acesso às principais publicações do mundo, as melhores credenciadas e aos hospitais também. Estão em constante contato com os hospitais que possuem serviços tecnicamente avançados.

Outro entrevistado comenta sobre a atualização de conhecimentos:

\begin{abstract}
"Eu além de exercer a função de dosimetrista aqui na clínica também sou tecnóloga em radiologia e fiz um mestrado em proteção radiológica com o intuito de aprimorar meu conhecimento, apesar de toda a equipe ser muito importante é técnico ou tecnólogo que efetivamente vai posicionar o paciente na posição correta, portanto considero a educação continuada desses profissionais fundamental para a reprodutibilidade do tratamento" E2
\end{abstract}

A fala anterior demonstra claramente a preocupação da dosimetrista, que é uma tecnóloga em radiologia, em se atualizar constantemente, inclusive fazendo um mestrado na área. Outra preocupação da profissional é a educação continuada dos técnicos e tecnólogos, pois a reprodutibilidade no tratamento de um paciente específico depende, em grande parte, do serviço desempenhado pelo técnico ou pelo tecnólogo.

Em Lages há um trabalho em conjunto com o Albert Einsten, onde realizam mesa redonda também quinzenal com a participação direta deste centro de referência. Apesar dos participantes consideraram as reuniões fundamentais para aprender, relataram que a leitura é a base para riqueza das discussões. O rádio-oncologista complementa: 
“Todos tem que estudar e ler, mas o médico principalmente, eu fui professor por 37 anos na federal e participo de uma serie de cursos, sou coordenador da liga de oncologia da Unisul e estou sempre envolvido com o ensino médico". E1

Hoje percebe-se que está é uma exigência, uma exigência funcional. Na radioterapia, grande parte das publicações são em inglês, pois está é a língua de comunicação cientifica do mundo. Então o tecnólogo tem a mesma obrigação de buscar informação o tempo todo, o enfermeiro tem a mesma obrigação de buscar o tempo todo. Ficou claro na fala dos participantes a importância de estudar continuamente e, a partir do momento que o profissional não estuda, ele irá perder autonomia, alguém vai dizer o que ele deve fazer.

Estamos vivenciando a sociedade do conhecimento, o conhecimento, está disponível para formatar o pensamento e pensamento é ação. Segundo Drucker (1999) [18], conhecimento é informação em efetiva para alcançar resultados. Na mesma linha, Polanyi (1966) [19] enfatiza a ação, o corpo e o conhecimento tácito, definindo conhecimento como a capacidade de agir.

É importante salientar que a renovação dos funcionários que trabalham no local pesquisado é muito baixa, o local possui funcionários com 30 anos de casa. As trocas são raras, pois quem trabalha no local possui um conhecimento agregado que é importantíssimo, com conhecimento e experiência que foram adquiridos ao longo dos anos participando de reuniões, lendo e aprendendo com os colegas. Isso confirma a pesquisa de Dorow (2017) [17] que demonstra a maior facilidade que grupos que interagem frequentemente em um contexto específico possuem para compartilhar conhecimento tácito.

Todos os funcionários são estimulados a buscar conhecimento. Existem vários casos de sucesso, como o da dosimetrista tecnóloga em radiologia com mestrado, e outros que estão sendo estimulados a se especializarem. Todos buscam especializações e o local incentiva e facilita a participação em congressos e eventos afins. O grupo pesquisado também compartilha conhecimento de forma significativa e colaborativa, a maioria das interações para compartilhar são face a face, o que influencia um ambiente propício ao compartilhamento do conhecimento corroborando a pesquisa de Dorow (2017) [17].

Todos participantes consideraram a reunião quinzenal a melhor forma de compartilhar conhecimento, principalmente devido ao aprofundamento das questões que são tratadas nesse tipo de discussão quando comparadas as interações realizadas no dia a dia são mais superficiais devido ao limite de tempo e agenda de trabalho. Foi identificado o uso maciço de algumas práticas de compartilhamento do conhecimento como revisão por pares, mentoring e storytelling.

A revisão por pares estava enraizada na cultura dos funcionários e se trata da revisão de um trabalho por outro profissional, seja para tirar uma dúvida ou somente quando o objetivo é uma segunda opinião. Serve principalmente para fornecer feedback e essa prática ocorria principalmente nas interações que permeiam a rotina de trabalho dos profissionais.

$\mathrm{O}$ mentoring foi identificado entre diversos profissionais, principalmente entre a dosimetrista, os médicos e físicos. Isso se deve ao fato de que o desenvolvimento da competência da dosimetrista ocorreu dentro da própria organização com o aprimoramento de seu conhecimento ao longo dos anos por meio da mentoria dos mais experientes. Essa prática implica em um especialista (mentor) modelar as competências de outro indivíduo, ou mesmo de todo grupo, observar, analisar o desempenho e fornecer feedback sobre as atividades desse indivíduo ou grupo [17]. Essa prática também ajudou a estreitar as relações entre os profissionais e acelerar a aprendizagem daqueles menos experientes.

As lições apreendidas, realizadas por meio de encontros para discussão permitiram não só o compartilhamento do conhecimento, mas também a criação de significado e identidade ao grupo ao promoverem um momento no qual os diferentes profissionais se sentiram seguros e confiantes para explicitar seu conhecimento.

A prática de storytelling serviu como veículo de conhecimento, pois se trata de histórias (casos clínicos), experiências, cultura e valores que são compartilhados entre os profissionais para facilitar a aprendizagem. O storytelling é aplicado no local pesquisado como uma prática altamente valiosa para o compartilhamento do conhecimento, o desenvolvimento de competências e a aprendizagem organizacional. Essa prática ocorreu principalmente nas reuniões de grupo e ajudava na criação de condições de aprendizagem e dinâmicas interativas para que os participantes pudessem 
compartilhar conhecimentos e experiências e aplicar os conceitos discutidos na reunião. Esta situação corrobora com diversas pesquisas que afirmam que as pessoas devem externalizar o seu conhecimento implícito fazendo uso da linguagem e do diálogo, o que lhes permite criar significado e interpretação para definir o contexto e as histórias facilitam muito esse processo [20, 21, 22, 23].

\section{CONCLUSÃO}

A radioterapia é um processo médico muito complexo que pode expor os pacientes a riscos indesejados devido a vários fatores. Em primeiro lugar, os pacientes que se submetem a um tratamento de radioterapia são geralmente afetados pelo câncer e, por essa razão, estão frequentemente em condições físicas e emocionais críticas.

O compartilhamento do conhecimento na radioterapia, especificamente, na teleterapia, foco deste estudo, é caracterizado por uma interação profunda entre funções totalmente automatizadas, fornecidas por equipamentos e tecnologias avançadas de hardware, software, combinado ao trabalho colaborativo de uma equipe formada por diferentes profissionais que realizam atividades interligadas que envolvem a tomada de decisão. Para que o tratamento do paciente seja possível, muitos profissionais de saúde devem estar envolvidos e este trabalho demonstrou que a comunicação entre esses profissionais é vital para o sucesso do que é planejado.

Diante do exposto, o objetivo desta pesquisa foi analisar o compartilhamento do conhecimento na melhoria da dinâmica organizacional em um serviço de teleterapia. Percebeu-se em relação ao serviço estudado um eficiente compartilhamento do conhecimento, fruto da frequência de interações entre os membros do grupo, do contexto específico de trabalho e do comprometimento dos profissionais de saúde que integram a equipe multidisciplinar, isso ajuda na geração de valor da organização. Ou seja, a cultura de zelar pela melhoria dos processos e pela boa comunicação da equipe diminui as chances de falhas na comunicação entre os membros da equipe, consequentemente, as chances de erro relacionadas ao tratamento.

No local pesquisado, a importância de um compartilhamento do conhecimento adequado faz parte da cultura da organização. Foi possível perceber que não basta a organização somente utilizar equipamentos e softwares modernos, é preciso que exista uma cultura que motive as pessoas a se comunicarem e compartilharem seus conhecimentos visando a qualidade de vida do paciente oncológico, e isso pode evidenciado na fala de todos os participantes da pesquisa, uma real e verdadeira preocupação com a melhoria de todos processos envolvidos e com a precisão do tratamento.

Na teleterapia uma filosofia multidisciplinar para diagnóstico e tratamento é a ferramenta para garantir a melhor definição do plano de tratamento, prevenir e aliviar os efeitos adversos do tratamento com o objetivo final de melhorar a sobrevida do paciente. Sendo assim, o conhecimento é um recurso muito valorizado pela equipe no planejamento e na execução de ações inteligentes relacionadas ao tratamento. As principais práticas de compartilhamento do conhecimento identificadas que permitem que a estratégia de tratamento do paciente seja compreendida por todos participantes da equipe multidisciplinar foram: a) revisão por pares; b) mentoring; e c) storytelling. Também se realizam reuniões para debater soluções clínicas, dúvidas e cuidados com o paciente oncológico. Mas além de tudo isso, o feedback sobre o processo de trabalho entre os membros da equipe multidisciplinar é dinâmico, fazendo com que o processo de aprendizagem da equipe seja contínuo.

Além disso, não existe uma cultura de punição e os erros são utilizados como oportunidades de aprendizagem para todo o grupo, isso garante um clima saudável na organização e um sentimento de grupo entre os envolvidos, onde todos se percebem como parte do "todo" e existe a consciência de que o papel de cada um é de extrema importância para o correto andamento do tratamento. Ficou notória a importância da equipe multidisciplinar e do conhecimento desses profissionais na garantia do tratamento seguro do paciente.

Conclui-se que o compartilhamento do conhecimento auxilia na melhora da dinâmica organizacional como um recurso estratégico, pois facilita o fluxo de conhecimento entre os membros da equipe e permite a reprodutibilidade diária do tratamento. 


\section{AGRADECIMENTOS}

Agradecemos ao local do estudo por abrir suas portas para esta pesquisa e aos professores do Instituto Federal de Santa Catarina que orientaram este trabalho.

\section{REFERÊNCIAS BIBLIOGRÁFICAS}

1. Okuno E. Radiação: efeitos, riscos e benefícios. São Paulo: Oficina de Textos; 2018. 144p.

2. Joana GS, Nogueira MS, Sandrini E, Bittencourt G, Salmon H, Fairbanks L, Gonçalves. M. Radiation therapy facility risk analysis in Brazil with SEVRRA software. J Radiol Prot. 2018;38(3):1128-1139, doi:10.1088/1361-6498/aad919

3. Hamilton DW, Heaven B, Thomson RG, Wilson JA, Exley C. Multidisciplinary team decision-making in cancer and the absent patient: a qualitative study. BMJ Open. 2016;1(6):1-8,doi:10.1136/bmjopen-2016012559

4. Papakostidi A, Tolia M, Tsoukalas N. Quality assurance in Health Services: the paradigm of radiotherapy. JBUON. 2014;19(1):47-52.

5. Cruz SG, Ferreira MMF. Gestão do conhecimento em instituições de saúde portuguesas. Rev Bras Enferm. 2016;69(3):461-8, doi:10.1590/0034-7167.2016690311i

6. Gider O, Ocak S, Top M. Perceptions of physicians about knowledge sharing barriers in Turkish health care system. J Medical Syst. 2015;39(42):41-42, doi:10.1007/s10916-015-0230-6

7. Brunner M, Gore SM, Read RL, Alexander MD, Mehta A, Elliot M, Milross C, Boyer M. Head and neck multidisciplinary team meetings: effect on patient management. Head Neck. 2015;37:1046-50. doi:10.1002/hed.23709

8. Karamitri I, Talias MA, Bellali T. Knowledge management practices in healthcare settings: a systematic review. Int J Health Planning Manage. 2017;32(1):4-18, doi:doi.org/10.1002/hpm.2303

9. Velloso MCM, Franklin MA, Daguano PZ, Simaro PA, Viersa CW, Riboldi PR. Gestão do conhecimento aplicado a serviços hospitalares: um estudo empírico em um hospital privado. FACEF PesquisaDesenvolvimento e Gestão. 2015 Mai;18(2):149-166.

10. Bardin L. Análise de Conteúdo. São Paulo: Edições; 2016. 70p.

11. Silva CM. Análise da movimentação da próstata com fiduciais na radioterapia guiada por imagem. [Dissertação]. Florianópolis (SC): Instituto Federal de Educação, Ciência e Tecnologia de Santa Catarina; 2017. 144.

12. Wang S, Noe RA. Knowledge sharing: A review and directions for future research. Human Resour Manage Rev. 2010 Jan;20(2):115-131, doi:10.1016/j.hrmr.2009.10.001

13. Hegenberg L. Saber de e saber que: Alicerces da racionalidade. Petrópolis: Vozes; 2002.

14. Nambisan S. Designing virtual customer environments for new product development: Toward a theory. Acad Manage Rev. 2002;27(3):392-413.

15. Rissi M. A confiança e as relações interpessoais assegurando o compartilhamento do conhecimento no ambiente virtual de aprendizagem. [tese]. Florianópolis (SC): Universidade Federal de Santa Catarina; 2013. $204 \mathrm{p}$.

16. Lindner F, Wald A. Success factors of knowledge management in temporary organizations. International J Project Manage. 2011;29(7):877-888. doi:10.1016/j.ijproman.2010.09.003

17. Dorow PF. Compreensão do compartilhamento do conhecimento em atividades intensivas em conhecimento em organizações de diagnóstico por imagem. [tese]. Florianópolis (SC): Universidade Federal de Santa Catarina; 2017. 361p.

18. Drucker PD. Desafios gerenciais para o século XXI. São Paulo: Pioneira; 1999. 120 p.

19. Polanyi M. The logic of tacit inference. Cambridge: Philosophy; 1966. 18p.

20. Batista F.F, Quandt CO, Pacheco FF, Terra JCC. Gestão do conhecimento na administração pública. Brasília: Instituto de Pesquisa Aplicada; 2005. 122p.

21. Chan WC, Jackson G, Wright CS, Orr-Walker B, Drury PL, Boswell DR, Jackson R. The future of population registers: linking routine health datasets to assess a population's current glycaemic status for quality improvement. BMJ Open. 2014 Abr;4(4), doi:10.1136/bmjopen-2013- 003975

22. Tsoukas H, Chia R. On organizational becoming: Rethinking organizational change. Org Science. 2002 Sep;13(5):567-582.

23. Dorow PF, Medeiros CD, Camozzato TSC, Silva C, Vargas FCD, Huhn A. Knowledge sharing process between radiologist. Int J Adv Res. 2018 Jul;6(7):431-436, doi:doi:0.21474/IJAR01/7380 